\title{
Reflets
}

Revue ontaroise d'intervention sociale et communautaire

\section{Le Groupe de recherche sur l'évaluation des troubles de la communication : historique, objectifs et réalisations}

JoAnne Paradis, Alain Desrochers et Linda Garcia

Volume 8, numéro 1, printemps 2002

La réadaptation : son visage français en Ontario

URI : https://id.erudit.org/iderudit/026373ar

DOI : https://doi.org/10.7202/026373ar

Aller au sommaire du numéro

Éditeur(s)

Reflets : Revue ontaroise d'intervention sociale et communautaire

ISSN

1203-4576 (imprimé)

1712-8498 (numérique)

Découvrir la revue

Citer cet article

Paradis, J., Desrochers, A. \& Garcia, L. (2002). Le Groupe de recherche sur l'évaluation des troubles de la communication : historique, objectifs et réalisations. Reflets, 8(1), 61-72. https://doi.org/10.7202/026373ar

Tous droits réservés (C) Reflets : Revue ontaroise d'intervention sociale et communautaire, 2002
Ce document est protégé par la loi sur le droit d'auteur. L'utilisation des services d'Érudit (y compris la reproduction) est assujettie à sa politique d'utilisation que vous pouvez consulter en ligne.

https://apropos.erudit.org/fr/usagers/politique-dutilisation/ 


\section{Le Groupe de recherche sur l'évaluation des troubles de la communication : historique, objectifs et réalisations}

\section{JoAnne Paradis}

Centre national de formation en santé pour francophones (CNFS)

Programme d'audiologie et d'orthophonie,

École des sciences de la réadaptation, Université d'Ottawa

\section{Alain Desrochers}

École de psychologie, Université d'Ottawa

\section{Linda Garcia}

Programme d'audiologie et d'orthophonie,

École des sciences de la réadaptation, Institut sur la santé des personnes âgées de l'Université d'Ottawa

\section{Introduction}

La présente édition de Reflets vise à dresser un bilan des services et des programmes de recherche qui s'inscrivent dans le secteur de la réadaptation communautaire en Ontario français. L'orthophonie constitue l'une des spécialités de la réadaptation. Les membres de cette profession étudient, évaluent et assurent la prise en charge des troubles de la communication humaine. Ainsi, les orthophonistes participent, entre autres, au diagnostic médical en ciblant spécifiquement les troubles de la voix, de la parole et du 
langage. Ces troubles peuvent affecter les locuteurs de tout âge, le langage parlé autant que le langage écrit. Dans certains cas, il est possible de déterminer que le désordre a une origine congénitale (p. ex., les fissures labiale ou palatine) ou qu'il résulte d'une lésion cérébrale (p. ex., l'aphasie); dans d'autres cas, l'origine du trouble est mal connue (p. ex., la dyslexie développementale). Dans cet article, nous nous proposons de décrire les activités d'un groupe de recherche qui s'intéresse précisément à l'évaluation des troubles de la communication et aux moyens disponibles pour mener cette opération auprès des Canadiens et Canadiennes d'expression française.

\section{Origine du GRETCOM}

Le Groupe de recherche sur l'évaluation des troubles de la communication (GRETCOM) ${ }^{1}$ a été fondé en 1995 par Alain Desrochers et Linda Garcia. Il s'agit d'une équipe de spécialistes en orthophonie et en psycholinguistique dont les intérêts gravitent autour de l'évaluation des troubles de la communication et des processus perceptivo-cognitifs qui président au langage tant oral qu'écrit, à la parole et à l'organisation du discours. Cette équipe comprend présentement trois membres :Alice Eriks-Brophy,Linda Garcia et JoAnne Paradis. Elle est affiliée au Programme d'audiologie et d'orthophonie de l'École des sciences de la réadaptation et à l'Institut sur la santé des personnes âgées, ces entités étant rattachées à l'Université d'Ottawa. Les membres du GRETCOM ont des expertises différentes, mais complémentaires. Par exemple, Alice Eriks-Brophy possède une expertise en évaluation et en intervention auprès des enfants atteints de troubles du langage ou d'une déficience auditive. Elle étudie les conséquences de ces difficultés sur l'intégration en milieu scolaire ainsi que les effets du multiculturalisme sur l'évaluation. Linda Garcia travaille sur l'intégration communautaire des adultes ayant des troubles de la communication et, particulièrement ceux qui ont une origine neurologique (p. ex., l'aphasie). Enfin, JoAnne 
Paradis s'intéresse à l'évaluation des adultes atteints de troubles de la parole et de la phonation d'origine neurologique ou consécutifs à un cancer cervico-facial.

\section{Objectifs du GRETCOM}

«Si le but de

l'évaluation consiste à

établir les niveaux de

performance

maximaux dans les

diverses fonctions de la communication, il faut mener cette opération dans la langue dans laquelle le patient se sent le plus compétent.»
Les objectifs du GRETCOM s'appuient sur un impératif :évaluer un patient dans sa langue dominante. Si le but de l'évaluation consiste à établir les niveaux de performance maximaux dans les diverses fonctions de la communication, il faut mener cette opération dans la langue dans laquelle le patient se sent le plus compétent. Pour les Canadiens et Canadiennes d'expression française, cela signifie que les mesures de performance doivent généralement être prises en français.

Les mesures de performance sont typiquement fournies par des batteries de tests ou des grilles d'observation. Ces mesures peuvent servir à : déterminer s'il existe un trouble et, le cas échéant, identifier la nature de ce trouble; isoler les facteurs qui ont pu entraîner ou maintenir le trouble observé; identifier des indices susceptibles de guider l'intervention ou estimer les effets d'une intervention (Garcia \& Desrochers 1997). À l'analyse, on a vite fait de constater que la communication humaine s'appuie sur une grande variété de fonctions. Les fonctions perceptives assurent le traitement des informations sur le plan sensoriel et leur intégration gnosique. Les fonctions langagières, au sens large, prennent en charge les traitements lexical, grammatical,sémantique et pragmatique. Enfin, les fonctions expressives président aux réalisations praxique et motrice. Un niveau de complication s'ajoute à l'évaluation des troubles de la communication lorsque des fonctions connexes au langage présentent un déficit (p. ex., les troubles de la mémoire). Le jeu complexe des fonctions qui assurent la communication exige une utilisation stratégique des outils d'évaluation dans la vérification des hypothèses cliniques (Nation \& Aram 1991). Cet impératif met en relief la pertinence de s'interroger sur l'état des outils d'évaluation en français. 
Le GRETCOM poursuit présentement deux objectifs généraux. Le premier consiste à identifier les ressources afférentes à l'évaluation des troubles de la communication auprès des Canadiens et Canadiennes d'expression française. Nous distinguons ici deux types de ressources : les ressources humaines, c'està-dire les personnes habilitées à effectuer des évaluations en français au Canada et les outils d'évaluation disponibles en français. Le deuxième objectif est de faire une appréciation de l'état actuel des ressources et d'évaluer leur utilité réelle dans la pratique de l'évaluation des troubles de la communication. Quelle est la répartition géographique des spécialistes de l'évaluation en français au Canada? Dans quel état d'adéquation se trouvent les outils d'évaluation en français à l'usage des spécialistes? Dans la prochaine section, nous ferons un bilan synthétique des réalisations du GRETCOM relativement à ces objectifs.

\section{Réalisations du GRETCOM}

\section{Les ressources humaines}

L'évaluation des troubles de la communication n'est pas l'affaire que d'une seule profession. Plusieurs groupes de spécialistes revendiquent leur part de marché : les orthophonistes, les audiologistes, les psychologues, les neuropsychologues, les éducateurs spécialisés, les orthopédagogues, etc. Il reste que la plupart des praticiens sont des orthophonistes, une main d'œuvre majoritairement féminine. Pour répertorier les personnes habilitées à effectuer des évaluations de la communication en français au Canada, nous avons mis en œuvre deux stratégies. Dans un premier temps, nous avons consulté l'annuaire des associations et des ordres professionnels d'orthophonistes-audiologistes au Canada et repéré les cliniciens qui se sont déclarés capables de conduire une évaluation en français. L'efficacité de cette stratégie est assurée par le fait que l'adhésion à un ordre professionnel est obligatoire pour les orthophonistes dans certaines provinces canadiennes ( $p$. ex., en Ontario). Dans un deuxième temps, nous avons recherché 
"Nous n'avons encore repéré aucun regroupement pan-canadien de spécialistes dont le mandat était spécifiquement d'apporter un appui à la pratique de l'évaluation des troubles de la communication en français.» les spécialistes qui annoncent leurs services sur le réseau Internet. En groupant les résultats de ces recherches, nous avons identifié plus de 1,200 spécialistes actifs au Canada dont 73\% oeuvrent au Québec, $17 \%$ en Ontario et 4\% au Nouveau-Brunswick.

Nous n'avons encore repéré aucun regroupement pancanadien de spécialistes dont le mandat était spécifiquement d'apporter un appui à la pratique de l'évaluation des troubles de la communication en français. Toutefois, le Regroupement des orthophonistes et audiologistes travaillant en français en Ontario (RAOFO) diffuse à ses membres, par le biais de son bulletin, des renseignements sur la pratique de l'évaluation et les outils disponibles en français. Le constat général que nous faisons, à ce stade, est que le réseau des spécialistes de l'évaluation des troubles de la communication en français au Canada est fragmenté et relativement fragile. L'état actuel du réseautage permet difficilement une mobilisation optimale des spécialistes et offre une protection réduite contre la duplication inutile des efforts pour améliorer la pratique de l'évaluation des troubles de la communication en français.

\section{Les outils d'évaluation}

L'arsenal des outils d'évaluation de l'orthophoniste doit être suffisamment diversifié pour assurer l'évaluation des troubles particuliers de la voix, de la parole et du langage. Comme Harris et Skaife Shelton (1993) de même que Shipley et McAfee (1992) l'ont fait pour les outils disponibles en anglais, le GRETCOM s'est donné comme but de répertorier tous les outils disponibles en français. Le premier constat que nous faisons est qu'il existe des outils en français pour conduire une évaluation de la plupart des troubles de la communication. Il se trouve, toutefois, que plusieurs de ces outils ont été élaborés en France ou en Belgique et qu'ils sont peu connus et à peu près inutilisés au Canada. L'impopularité de ces outils au Canada nous semble liée aux marchés visés par leurs concepteurs et, indirectement, au fait qu'ils paraissent souvent mal adaptés au contexte culturel canadien. Il 
"Quels sont les outils d'évaluation en français utilisés le plus fréquemment par les orthophonistes? À quelles difficultés sontelles confrontées dans leur utilisation?» existe présentement peu d'outils d'évaluation conçus au Canada, pour les Canadiens et Canadiennes d'expression française et appuyés par un réseau de distribution commerciale. Nous avons noté cependant l'utilisation de traductions ou d'adaptations françaises de tests américains dans la pratique de l'orthophonie au Canada français, peu importe si les spécialistes sont à l'œuvre dans une province ou une collectivité où le français est la langue majoritaire ou minoritaire. Les problèmes soulevés par ces traductions sont essentiellement de deux types. D'une part, les références culturelles ne s'accordent pas toujours avec le vécu des Canadiens et Canadiennes. D'autre part, les critères de compétence langagière choisis par les concepteurs sont nécessairement guidés par les habiletés jugées essentielles pour communiquer efficacement en anglais. Les particularités du français qui ne sont pas présentes dans la langue anglaise sont ipso facto exclues de ces tests. Cela dit, il existe, dans l'ensemble des outils d'évaluation, un problème plus important encore et de plus haut niveau :l'absence presque complète de standardisation des tests et de cadre de référence normatif pour l'interprétation des résultats. En dépit, des obstacles que nous avons pu identifier, et dont les conséquences seront discutées plus bas, il nous a été possible de dresser une liste substantielle d'outils d'évaluation en français que nos lecteurs pourront télécharger du site Internet du GRETCOM.

Les renseignements que nous avons rassemblés jusqu'à présent nous ont permis d'établir la liste des outils que les spécialistes de l'évaluation des troubles de la communication pourraient utiliser dans leur pratique. Mais il nous manque un renseignement important pour compléter le bilan que nous tentons de dresser : les choix opérés par les personnes concernées. Quels sont les outils d'évaluation en français utilisés le plus fréquemment par les orthophonistes? À quelles difficultés sont-elles confrontées dans leur utilisation? Pour nous faire une idée plus juste de l'usage des outils, nous lançons une enquête pan-canadienne auprès des orthophonistes. Une enquête similaire menée par Huang, Hopkins et Nippold (1997) aux États-Unis nous a servi de modèle. Cette recherche, présentement en cours, vise d'abord à mieux connaitre les caractéristiques des orthophonistes habilitées à conduire une 
évaluation en français : leur formation, leur expérience professionnelle et leur expertise, leurs langues de travail, leur province ou territoire de prestation de service, leur milieu de travail, leurs heures de travail, la proportion des heures consacrée à l'évaluation, les tranches d'âge de leur clientèle. Nous sondons également les usages qui sont faits des outils d'évaluation, les renseignements techniques (p. ex., les indices dérivés des résultats, les propriétés psychométriques des instruments) jugés utiles par les spécialistes, leur degré de satisfaction relatif à divers aspects de l'évaluation et aux outils disponibles en français ainsi que les solutions mises en œuvre pour assurer la justesse ou l'utilité de leurs évaluations. Enfin, nous leur demandons de rapporter les cinq outils qu'elles utilisent le plus fréquemment pour évaluer chacun d'une série de troubles de la communication. Un compte rendu sommaire de cette enquête pourra être téléchargé du site Internet du GRETCOM dans les prochains mois.

Notre démarche ne s'arrête pas à dresser un bilan des outils d'évaluation disponibles en français ou à identifier ceux qui sont le plus fréquemment utilisés. Nous menons, en parallèle, une analyse fine des attributs de ces outils. Cette analyse s'appuie sur plusieurs critères : un schème de classement qui met en relief les fonctions de la communication et la zone d'âge visées par le test; la standardisation du test, son cadre de référence normatif et les propriétés psychométriques des mesures qui en résultent et des considérations pratiques telles que le temps de passation, le matériel requis et la facilité d'administration. Cet exercice fournit une base pour identifier les meilleurs instruments sur le marché et ceux qui mériteraient l'attention des chercheurs intéressés à leur apporter des améliorations.

\section{Les retombées}

Le port d'attache du GRETCOM se trouve en Ontario, mais ses activités visent toute la francophonie canadienne. Les difficultés que nous avons décelées dans la pratique de l'orthophonie en 
français ne sont pas propres à une province ou à une région; elles sont essentiellement les mêmes pour tous les praticiens. Examinons maintenant les répercussions attendues des réalisations récentes du GRETCOM ou des projets en cours.

\section{L'accès à l'information}

Outre les publications dans des périodiques, le moyen le plus efficace que nous avons trouvé pour assurer l'accès à l'information relative à l'évaluation des troubles de la communication est le réseau Internet. Les analyses et les répertoires que nous préparons pourront être téléchargés du site Internet du GRETCOM : www.gretcom.uottawa.ca. Ce moyen de communication offre la même accessibilité aux spécialistes qui travaillent en milieu urbain qu'à ceux dans les régions éloignées des grandes villes.

Le répertoire des outils d'évaluation que nous assemblons permettra aux spécialistes de l'évaluation de la communication de prendre connaissance des ressources couramment disponibles en français et, nous l'espérons, d'améliorer les services offerts à la clientèle d'expression française. Ces renseignements seront utiles aux praticiens ainsi qu'aux enseignants et superviseurs de stages cliniques soucieux de diriger leurs étudiants vers les meilleurs outils de travail sur le marché. Sur le plan de la recherche, les chercheurs auront accès à des outils standardisés qui leur permettront de mieux sélectionner leurs sujets francophones. Les résultats de l'enquête pan-canadienne auprès des spécialistes de l'évaluation en français fourniront à tout internaute une vue sur l'état ponctuel de la profession et une liste des outils de travail couramment utilisés. Ces renseignements pourront être particulièrement utiles aux jeunes professionnels et aux décideurs chargés de l'achat des tests dans un établissement scolaire ou hospitalier. Les ressources accessibles par le site Internet du GRETCOM sont encore limitées, mais il faut prévoir que leur nombre et leur diversité augmenteront au fur et à mesure que 
nos projets de recherche et ceux de nos partenaires arriveront à terme.

Finalement, l'accès à l'information permettra un échange fructueux entre cliniciens francophones travaillant auprès de populations similaires ou encore entre cliniciens et chercheurs voulant collaborer sur des protocoles de recherche.

\section{Une analyse du rendement des outils d'évaluation}

L'analyse fine des outils d'évaluation en français nous a permis d'identifier deux types de risques dans leur utilisation. Le premier porte sur l'erreur dans l'évaluation des hypothèses cliniques. Pour identifier un trouble de la communication, on doit généralement se rapporter au niveau de performance normatif (ou normal) dans la population de référence. Sans cette norme, il est difficile de faire une appréciation précise du degré de déviation de la performance d'un individu par rapport à celle de son groupe de référence. Or, il se trouve que le cadre de référence normatif et les indices dérivés des scores aux tests (p. ex. le rang centile) sont souvent absents des outils d'évaluation en français. Les professionnels de l'évaluation pallient ce problème en superposant aux scores un cadre de référence implicite issu de leur expérience clinique. Il reste que l'élaboration de cadres de référence normatifs appropriés, un des objectifs du GRETCOM, augmenterait considérablement la précision de l'évaluation et, du même coup, réduirait le risque d'une erreur dans la vérification des hypothèses cliniques, particulièrement chez les jeunes professionnels.

Lorsque le cadre de référence normatif d'un test n'est pas établi, il se trouve aussi que la marge d'erreur de la mesure n'est généralement pas connue. Cette situation a une conséquence immédiate sur notre capacité à évaluer l'effet d'un traitement. Il devient alors périlleux de décider si une augmentation de la performance langagière résulte de l'effet du traitement ou d'une fluctuation aléatoire de la mesure. La difficulté d'établir au-delà de tout doute raisonnable la marge d'erreur de la mesure issue 
d'un outil d'évaluation et celle d'estimer le degré de déviation par rapport à un niveau de performance normal rendent très difficile l'élaboration d'une justification pour autoriser un traitement coûteux ou pour appuyer une preuve en cour de justice.

\section{Défis à relever}

L'analyse systématique des ressources dans une pratique professionnelle est une arme à deux tranchants. D'une part, elle peut permettre d'établir exhaustivement l'ensemble des moyens d'action à la disposition des praticiens. D'autre part, elle peut mettre en évidence ses lacunes, ses faiblesses et les risques que celles-ci entraînent. Il nous semble que le défi le plus urgent à relever dans la pratique de l'orthophonie en français est sa normalisation. Par normalisation de la pratique, nous entendons l'amélioration des outils d'évaluation existants, l'élaboration d'outils adaptés au milieu culturel canadien, l'établissement de cadres de référence normatifs représentatifs des populations d'expression française au Canada. Dans les travaux de recherche sur le Test de rendement pour francophones (1994), on a montré la nécessité d'établir des normes séparées pour les francophones résidant au Québec et à l'extérieur du Québec. Il pourrait en être de même pour plusieurs tests utilisés en orthophonie. Le deuxième défi consiste à démontrer la nécessité d'un appui financier soutenu auprès des conseils de recherche pour faire avancer les travaux sur l'amélioration et l'élaboration des outils d'évaluation de la communication.

\section{Conclusion}

Pour relever les défis dont nous venons de parler, il faut réunir plusieurs conditions. D'abord, il est impératif de dresser un bilan juste et précis de l'état de la pratique de l'évaluation des troubles 
"D'abord, il est impératif de dresser un bilan juste et précis de l'état de la pratique de l'évaluation des troubles de la communication en français, avec ses forces et ses faiblesses.» de la communication en français, avec ses forces et ses faiblesses. Ce bilan doit pouvoir guider efficacement les activités des chercheurs, des praticiens et des formateurs. Il permettra d'identifier les meilleurs moyens ponctuellement disponibles et ceux dont l'utilisation présente des risques excessifs dans la pratique de l'orthophonie. Le changement passe ordinairement par l'initiative individuelle, en recherche ou dans la pratique, mais l'effort est plus constant et soutenu lorsqu'il peut s'appuyer sur une infrastructure et un financement stable. L'élaboration rigoureuse d'outils d'évaluation des troubles de la communication est une activité coûteuse qui exige du temps et de la détermination de la part des chercheurs. Il est difficile de concevoir la mise en œuvre d'un changement réel sans l'appui soutenu des infrastructures de recherche au Canada. Il ne faut pas négliger non plus la mise en marché et la distribution des outils d'évaluation. Le moyen le plus efficace d'assurer la mise en marché et la distribution des outils d'évaluation aux spécialistes demeure le marché de l'édition. Le marché des tests en français étant restreint au Canada, il est impératif de mettre en œuvre des systèmes de production et de distribution économiques. Si les marges bénéficiaires sont insuffisantes, le marché des tests en français en orthophonie sera jugé non viable au Canada. Enfin, l'adoption d'un outil d'évaluation dans la pratique d'une profession dépend largement des formateurs, c'est-à-dire les enseignants et les superviseurs cliniques. Pour favoriser l'utilisation des meilleurs outils disponibles en orthophonie, il est impératif que leur valeur et leur utilité soit reconnues, attestées et véhiculées par les professionnels chargés de la formation des futurs spécialistes de l'évaluation des troubles de la communication. Nous croyons que les ressources individuelles et collectives de la profession sont considérables et qu'un déploiement bien structuré des efforts conduira à l'amélioration dans la qualité des services offerts aux Canadiens et Canadiennes d'expression française.

\section{Références}

GARCIA, Linda J. et Alain DESROCHERS (1997). «L'évaluation des troubles du langage et de la parole chez l'adulte francophone/Assessment of Language and Speech Disorders in Francophone Adults ", Journal of Speech-Language Pathology and Audiology, 21:4, 271-293. 
HARRIS, Lonnie G. et Ivy SKAIFE SHELTON (1993). Desk reference of assessments in speech-language. Tucson, AZ : Communication Skill Builders, Division of the Psychological Corporation.

HUANG, Rei-Jane, Joseph HOPKINS et Marilyn A. NIPPOLD (1997). "Satisfaction with standardized language testing :A survey of speech-language pathologists ». Language, Speech, and Hearing Services in Schools, 28, 12-29.

NATION, James E. et Dorothy M. ARAM (1991). Diagnosis of speech and language disorders. San Diego, CA : Singular Publishing Group, Inc.

SHIPLEY, Kenneth G. et Julie G. MCAFEE (1992). Assessment in speech-language pathology :A resource manual. San Diego, CA : Singular Publishing Group, Inc.

Test de rendement pour francophones (1994). Toronto :The Psychological Corporation.

\section{Note}

1. L'Université d'Ottawa fournit au GRETCOM son support Internet et lui permet de faire avancer des projets spécifiques en lui octroyant des subsides internes. L'équipe est présentement logée au Service de santé des Sœurs de la Charité d'Ottawa (SCO) et à l'Institut sur la santé des personnes âgées de l'Université d'Ottawa. Elle obtient la plus grande partie de son financement $\mathrm{du}$ Centre national de formation en santé pour francophones (CNFS) et du Regroupement des universités de la francophonie hors Québec (RUFHQ). Notre reconnaissance va également au Regroupement des audiologistes et des orthophonistes travaillant en français en Ontario (RAOFO) et aux autres professionnels de l'évaluation des troubles de la communication qui nous ont accordé leur appui.Toute correspondance relative à cet article devrait être adressée au Groupe de recherche sur l'évaluation des troubles de la communication (GRETCOM), 43 rue Bruyère, Ottawa (Ontario) Canada, K1N 5C8; téléphone : (613) 562-6039; télécopieur :(613) 562-6039; courriel : gretcom@scohs.on.ca; Site Internet : www.gretcom.uottawa.ca. 